\title{
Staged concept of laser-plasma acceleration toward multi-GeV electron beams
}

\author{
Victor Malka, A. Lifschitz, J. Faure, and Y. Glinec \\ LOA, ENSTA, X, CNRS, UMR 7639, 91761 Palaiseau, France
}

(Received 19 July 2006; published 12 September 2006)

\begin{abstract}
The concepts of the laser-plasma based accelerator and injector are discussed here. The recent tests done at LOA as well as design studies of high-quality $\mathrm{GeV}$ electron beam production with low energy spread $(1 \%)$ are presented. These laser-produced particle beams have a number of interesting properties and could lend themselves to applications in many fields, including medicine (radiotherapy), chemistry (radiolysis), and accelerator physics. They could be used as a source for the production of $\gamma$ ray beams for nondestructive material inspection by radiography, or for future compact $\mathrm{X}$-free electron laser machines.
\end{abstract}

DOI: $10.1103 /$ PhysRevSTAB.9.091301

PACS numbers: 41.75.Jv, 29.25.Bx, 29.27.Bd, 52.38.Ph

\section{INTRODUCTION}

High-gradient acceleration techniques rely on electron plasma waves to efficiently accelerate particles. The concept of laser-plasma acceleration was originally proposed 25 years ago by Tajima and Dawson [1]. The electric field in a plasma wave can attain extremely high values, of the order of $E_{0}=c m_{e} \omega_{p} / e, \quad E_{0}(\mathrm{~V} / \mathrm{m}) \gg 96 n_{e}\left(\mathrm{~cm}^{-3}\right)^{1 / 2}$ $\left(E_{0}=190 \mathrm{GV} / \mathrm{m}\right.$ for $\left.n_{e}=3.9 \times 10^{18} \mathrm{~cm}^{-3}\right)$. Plasma waves can be excited by the propagation of laser pulses in a transparent plasma, i.e., in plasmas with density below the critical density, $n_{c}\left(\mathrm{~cm}^{-3}\right)=1.1 \times 10^{21} / \lambda_{0}{ }^{2}(\mu \mathrm{m})$. In the scheme known as laser wakefield acceleration, the plasma waves are generated in the wake of the laser pulse, like waves in the wake of a ship. Efficient energy transfer between the wave and the particles requires that both move at the same speed. High energy gain can be reached by using low density plasmas, in which the phase velocity of the laser-excited plasma wave, which is equal to the laser pulse group velocity, is very close to the speed of light in vacuum. The longitudinal electric fields associated with this fast plasma wave is then able to accelerate relativistic particles injected into the plasma or even, if its amplitude is large enough, to trap particles from the plasma itself. By "surfing" on this electrostatic wave, particles can be boosted to high energies over very short distances.

Laser-plasma acceleration techniques have successfully been used either for acceleration of injected electrons or as a source of electrons. The later will be called "plasma injector."

Excitation of relativistic plasma waves by intense laser pulses is made possible by the ponderomotive force (related to the "light pressure"), which expels electrons from regions of high laser intensity gradient. Since this force is proportional to the laser intensity gradient, large amplitude plasma waves can be excited using relatively modest laser energies by using very short pulses. In the wake field regime, plasma waves can be excited efficiently when the laser pulse duration $\tau_{L}$ is close to 0.37 times the plasma period $\tau_{p}$. The plasma period is related to the electron density by $\tau_{p}=2 \pi / \omega_{p}$, with $\omega_{p}^{2}=n_{e} e^{2} / m_{e} \varepsilon_{0}$. The resonant condition in practical unit is then given by $n_{e}\left(\mathrm{~cm}^{-3}\right)=1.6 \times 10^{21} / \tau_{L}^{2}(\mathrm{fs})$, for a 30 fs laser, this gives a resonant density of $1.75 \times 10^{18} \mathrm{~cm}^{-3}$.

On the other hand, the phase velocity of the wake $v_{\text {plasma }}$, which is equal to the group velocity of the laser $v_{\text {glaser }}$, increases when the electron density decreases, indicating that higher maximum energy gain can be reached in lower density plasmas according to $v_{\text {plasma }}=v_{\text {glaser }}=$ $c\left(1-\omega_{p}{ }^{2} / \omega_{0}^{2}\right)^{1 / 2}=c\left(1-n_{e} / n_{c}\right)^{1 / 2}$.

Acceleration of injected electrons in laser-plasma wakefields has been successfully demonstrated using one laser beam in the laser wake field regime (LWF) or two lasers beams in the laser beat wave regime (LBW).

Electrons injected at $3 \mathrm{MeV}$ have been accelerated by this scheme up to $4.7 \mathrm{MeV}$ [2]. In the LBW, the plasma wave is driven by the interference of two laser pulses (not necessary short) at two different wavelengths. Electric fields close to the $\mathrm{GV} / \mathrm{m}$ level were measured for this experimental configuration using midinfrared laser beams [3] and injected electrons have been accelerated up to $30 \mathrm{MeV}$ at UCLA [4]. In both LWF and LBW, only electrons injected by an external source into the wave have been accelerated.

In those experiments, the injected electron beam always had a duration much greater than the plasma period and, as a consequence, electrons of the bunch experienced the whole sinusoidal electric field, and had an output spectra with a Maxwellian-like distribution. The efficient production of monoenergetic beams with plasma accelerating structures requires the development of bunches of electrons with durations much shorter than the plasma period, i.e., typically in order of $10 \mathrm{fs}$ or less to be accelerated in a constant electric field in a plasma wave structure with 100 fs period.

In homogenous plasmas, the interaction length is limited by the natural diffraction length, the Rayleigh length, given by the numerical aperture of the laser beam and its wavelength $\lambda_{0}$ :

$$
L_{\text {int }}=\pi Z_{R}, \quad \text { with } Z_{R}=\pi w_{0}^{2} / \lambda_{0},
$$


where $w_{0}$ is the laser focal spot waist. In the linear wakefield regime and at resonance, the plasma wave amplitude, $E_{\max } / E_{0}$ (or $\left.\delta n / n\right)$ is related to $a_{0}$, the laser strength parameter according to $E_{\max } / E_{0} \approx 0.38 \times a_{0}^{2}$, which is related to the laser peak intensity : $a_{0} \approx 8.6 \times$ $10^{-10}\left(I_{L} \lambda_{0}{ }^{2}\right)^{1 / 2}\left(I_{L}\right.$ and $\lambda_{0}$ are, respectively, expressed in $\mathrm{W} / \mathrm{cm}^{2}$ and in $\left.\mu \mathrm{m}\right)$. In order to get intense but linear plasma waves, $a_{0}$ must be of the order of 1 , in other words, the laser peak intensity (for $\lambda_{0}=800 \mathrm{~nm}$ ) must be of the order of $10^{18} \mathrm{~W} / \mathrm{cm}^{2}$. With current laser technology (in the level of tens of TW), a laser beam focused with an $f / 20$ aperture can generate a plasma wave in the linear regime $\left(a_{0} \sim 1\right)$ over an interaction length of a few millimeters. This value has to be compared to the dephasing length, which is the maximum length over which electrons are accelerated: $L_{d} \approx \gamma_{p}{ }^{2} \lambda_{p}$ where the relativistic factor of the plasma wave $\gamma_{p}=\left(1-v_{p}{ }^{2} / c^{2}\right)^{-1 / 2}$ is close to $\lambda_{p} / \lambda_{0}$ for tenuous plasmas. The plasma wavelength is related to the electron density by $\lambda_{p}(\mu \mathrm{m}) \approx 3.3 \times$ $10^{10} n_{e}\left(\mathrm{~cm}^{-3}\right)^{-1 / 2}$. For a $30 \mathrm{fs}$ laser pulse, the dephasing length, $L_{d} \approx \lambda_{p}{ }^{3} / \lambda_{0}{ }^{2}$, at the resonance is of the order of $23 \mathrm{~mm}$. The maximum energy gain $W_{\max } \approx$ $4 \gamma_{p}{ }^{2}\left(E_{\max } / E_{0}\right) m c^{2}$ is then given by or $W_{\max }(\mathrm{MeV}) \approx$ $0.77 n_{c} / n_{e} a_{0}^{2}$ which is close to $750 \mathrm{MeV}$. In the abovementioned experimental studies of acceleration of externally injected electrons, the energy gain was limited by the interaction length, which was much smaller than the dephasing length.

Since the physics of laser interaction with underdense plasma in the weakly relativistic regime is mainly governed by linear processes, the corresponding accelerating structures should have very good stability, only limited by the laser or target shot to shot fluctuations. The problem of stability in these schemes does not seem to be a real one for future accelerators due to the rapid progress in laser and target technologies. On the other hand, when the laser power exceeds the self-focusing power, plasma waves can reach very high levels and trap plasma electrons directly without external injection. Several experiments have been performed in these conditions, with laser powers in the range of TW and laser energies (and durations) in the range of $0.1 \mathrm{~J}(\sim 30 \mathrm{fs})$ to $100 \mathrm{~J}$ (ps). Those "plasma injectors" have been the subject of intense research efforts during these past years.

\section{LASER-PLASMA INJECTOR}

In 2002, the quality and the electron maximum energy were improved in experiments using a 30 fs laser and a low density plasma. By choosing a plasma density close to resonance, nonlinear plasma waves were excited permitting the production of electron energy distributions with a Maxwellian shape and with a plateau extending up to $200 \mathrm{MeV}$ [5]. The electron beam quality was enhanced due to the reduction of laser-electron beam interaction. For the above parameters, the electric field inside the plasma was estimated from PIC simulations which showed that it could reach a record value of more than $1.4 \mathrm{TV} / \mathrm{m}$. Under these extreme conditions, it takes less than $1 \mathrm{~mm}$ of laser propagation in the plasma to accelerate electrons up to $200 \mathrm{MeV}$.

The electron energy gain was improved and so was the emittance, which reached values comparable to those of modern conventional accelerators [6]. Whereas accelerated electron distributions previously suffered from a very large energy spread, a new and extremely exciting milestone has been reach recently, when three groups, from Imperial College [7], Lawrence Berkeley National Lab [8] (LBNL), and LOA [9], independently produced monoenergetic, high energy electron beams, directly by focusing a laser pulse onto an homogenous [7,9] or into a plasma channel [8]. The production of such beams had been predicted by numerical simulations [10].

Tests of the laser-plasma accelerator were made at LOA by focusing a $30 \mathrm{TW}$ laser with a $f / 18$ off-axis parabola onto a gas jet target. The focal spot had a diameter at full width half maximum (FWHM) of $18 \mu \mathrm{m}$ and the corresponding laser intensity is $I=3.2 \times 10^{18} \mathrm{~W} / \mathrm{cm}^{2}$. This choice of small aperture gives a long Rayleigh length of about $1.3 \mathrm{~mm}$, range of interest for higher energy gain as mentioned above. Measurements of the electron beam distribution, in energy and in space, are a powerful way to gain better understanding of processes involved in the laser-plasma interaction. We develop a new method based on the use of a compact spectrometer consisting of a $0.45 \mathrm{~T}$, $5 \mathrm{~cm}$ long permanent magnet and a LANEX phosphor screen. Doing so, we were able to measure, in a single shot, the whole spectra and to record its image on a 16 bit charged coupled device (CCD) camera. Since we do not

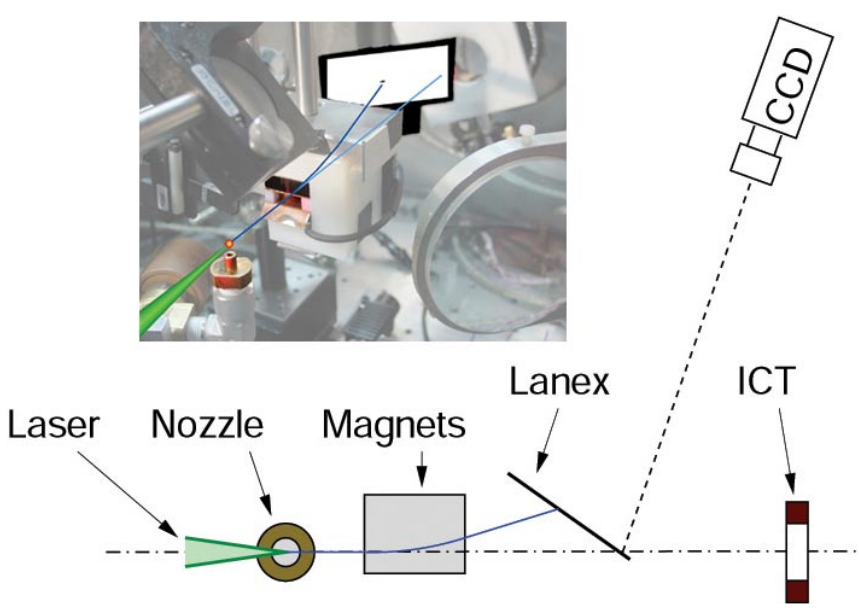

FIG. 1. (Color) Picture and layout of the experimental setup. The compact spectrometer consisting of a permanent magnet and a phosphor screen recorded the whole spectrum (above $20 \mathrm{MeV}$ ) on a 16 bit charged coupled device (CCD) camera. The integrator charge transformer (ICT) measures the charge of electrons with energies greater than $100 \mathrm{MeV}$. 
use a collimator, the vertical direction on the LANEX screen corresponds to the angular aperture of the electron beam. The LANEX screen was protected by a $100 \mu \mathrm{m}$ thick aluminum foil in order to avoid direct exposure to the laser light. The resolution was, respectively, 32 and $12 \mathrm{MeV}$ for 170 and $100 \mathrm{MeV}$ energies. The spatial electron beam distribution was measured by removing the magnet. The charge of the electron beam was measured using an integrating current transformer placed $30 \mathrm{~cm}$ behind the LANEX. The emission from the LANEX screen was also absolutely calibrated using a $10 \mathrm{MeV}$ rf accelerator [11]. The location of the ICT (after the magnet), allows the measurement of the charge of electrons with energies greater than $100 \mathrm{MeV}$. A schematic description and a picture of the inner vacuum chamber are presented in Fig. 1.

Figure 2 shows the electron spectra obtained in the optimal condition. At higher densities the spectrum has a Maxwellian-like distribution. This transition occurs for densities around $1.0 \times 10^{19} \mathrm{~cm}^{-3}$. The best coupling for obtaining a high charge and a quasimonoenergetic electron beam is at $n_{e}=6 \times 10^{18} \mathrm{~cm}^{-3}$. For this density, the image shows a narrow peak around $170 \mathrm{MeV}$, indicating efficient monoenergetic acceleration with a $24 \%$ energy spread (corresponding to the spectrometer resolution). The charge contained in this bunch can be inferred using the integrating current transformer: the whole beam contains $2 \pm$ $0.5 \mathrm{nC}$ and the charge at $170 \pm 20 \mathrm{MeV}$ is $0.5 \pm 0.2 \mathrm{nC}$. The electron beam energy was estimated to be close to $100 \mathrm{~mJ}$, which correspond to almost $10 \%$ of the laser energy. At lower density, $n_{e}=3 \times 10^{18} \mathrm{~cm}^{-3}$, the peak energy is at approximately the same position whereas the number of electrons is reduced by a factor of 10 . The spatial distribution of the electron beam is indicated also in Fig. 2 showing that the beam is collimated with a $6 \mathrm{mrad}$ divergence angle (at FWHM).

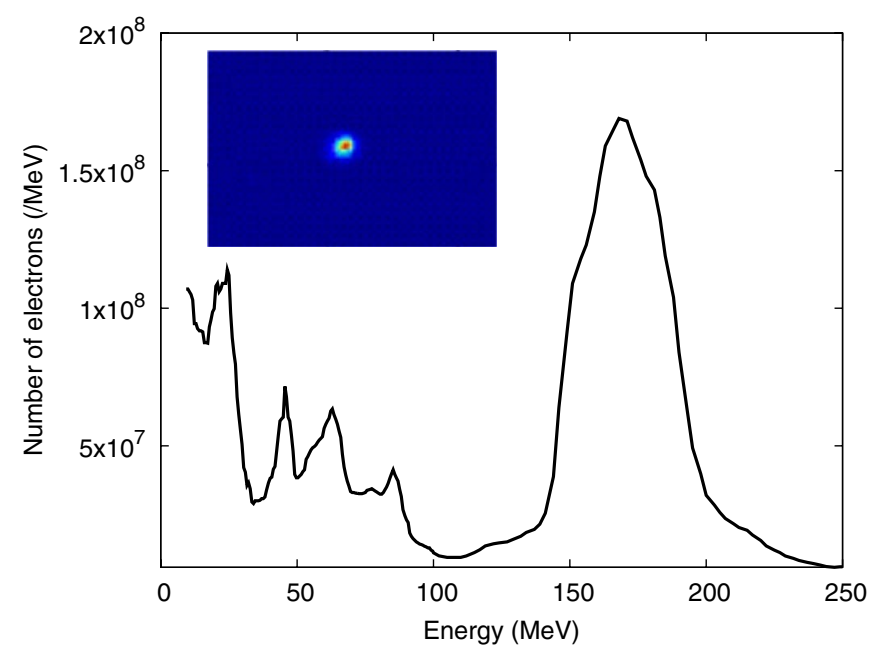

FIG. 2. (Color) Typical electron spectra obtained at $6 \times$ $10^{18} \mathrm{~cm}^{-3}$ with $1 \mathrm{~J}, 30 \mathrm{fs}$ laser pulse focused down a $18 \mu \mathrm{m}$ focal spot.
Similar results have also been achieved using a preformed plasma channel with a gas jet target [8]. Quasimonoenergetic beams have also been produced with homogenous or parabolic plasmas at higher density and even with longer laser pulse with $\tau_{L}>\tau_{\text {plasma }}$. In those cases, part of the laser pulse energy generated a bubble cavity which can sustain the self-injection and the production of quasimonoenergetic spectra. To optimize the energy transfer to the electron bunch, the laser pulse duration must be of the order of the plasma period. The optimal case corresponded to the plasma density $n_{e}=6 \times 10^{18} \mathrm{~cm}^{-3}$ [12].

The simulation reveals that the laser pulse self-focuses as it propagates in the plateau region of the gas jet. As the effective radius of the laser pulse decreases, the laser intensity increases and finally becomes sufficient to generate the so-called "bubble" [10], which is the shape of the plasma wave when the peak vector potential greatly exceeds unity. Since the laser pulse also pushes electrons forward, it propagates in a plasma with rising density. The laser group velocity will then be higher on the back of the pulse than on its front. This will compress temporally the laser pulse as was recently measured [13] and will also contribute to the generation of the bubble. The laser ponderomotive force expels the plasma electrons radially and leaves a cavitated region behind the pulse. When the beam charge becomes comparable with the ion charge in the cavity, the injection stops, which finally leads to a monoenergetic electron beam. Temporal information based on infrared emission measurements indicated that the electron bunch had temporal structures with durations shorter than 50 fs $[14,15]$.

\section{LASER-PLASMA ACCELERATORS}

It has been shown that the bubble or blow out regime can produce electron beams at high energy with an energy spread of about $5 \%$ to $10 \%$. Numerical simulations show that, with a $200 \mathrm{TW}$ laser, electron energies of $1.5 \mathrm{GeV}$ can be achieved in homogenous plasmas [16]. Using a plasma channel the laser power needed to reach similar gain is reduced to $50 \mathrm{TW}$ level [17]. Nevertheless, since the energy transfer from the laser to the electron beam is the same, one has to find a compromise between the charge and the energy. Typical laser to electron beam energy transfer is of the order of $10 \%$. Assuming this value and a final electron energy of $1.5 \mathrm{GeV}$, for a $200 \mathrm{TW}-50 \mathrm{fs}$ laser (i.e., $10 \mathrm{~J}$ ), the charge will be limited to $666 \mathrm{pC}$, whereas for a $50 \mathrm{TW}-50$ fs (i.e., $2.5 \mathrm{~J}$ ), the maximum charge will be $166 \mathrm{pC}$.

Here, we consider a two stage laser-plasma accelerator in the $\mathrm{GeV}$ range for which the relative energy spread can be decreased below $1 \%$. The second stage is an electricfield structure based on a laser wakefield. The generation of stable accelerating structures relies on the excitation of 


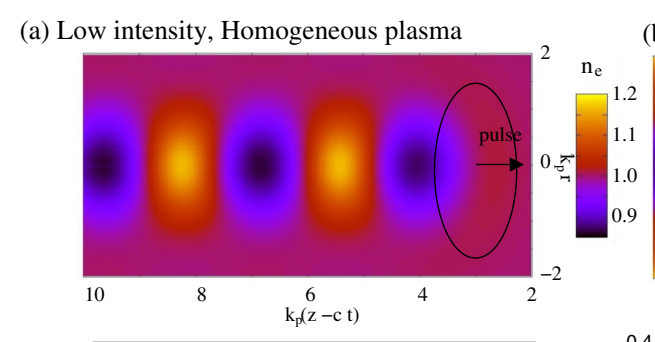

(b) Low intensity, Parabolic channel

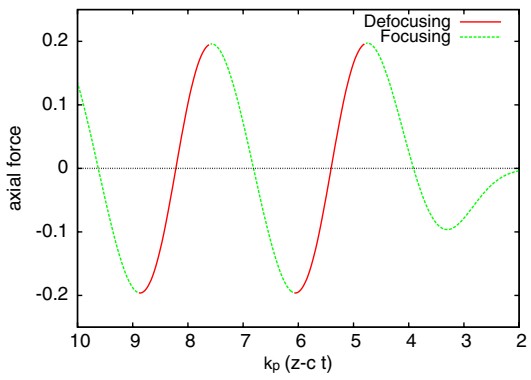

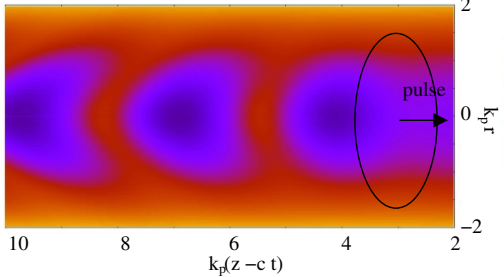

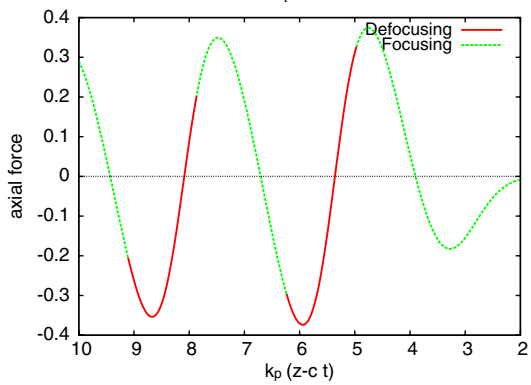

(c) Moderate intensity, Homogeneous plasma

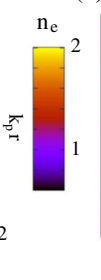

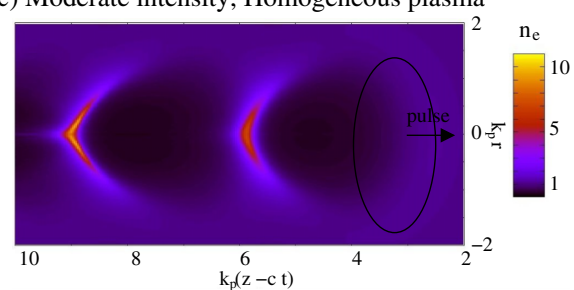

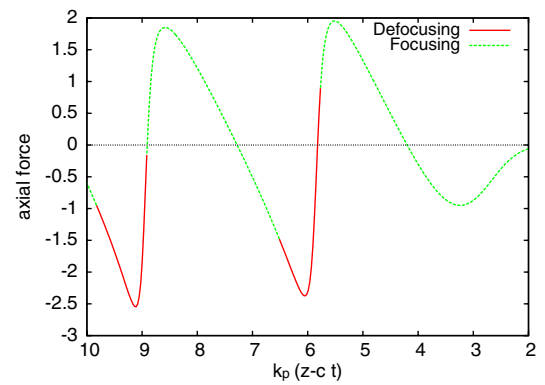

FIG. 3. (Color) Electron density spatial distribution $n_{e}(r, \xi)$ (top) and longitudinal force on electrons vs $\xi$ (bottom) for three laser and plasma conditions: (a) Low intensity pulse $\left(a_{0}=0.5\right)$ and homogeneous plasma, (b) low intensity pulse $\left(a_{0}=0.5\right)$ and parabolic plasma channel, and (c) moderate intensity pulse $\left(a_{0}=2\right)$ and homogeneous plasma.

moderate amplitude plasma waves driven by lasers with moderate laser amplitude.

We present first three scenarios for the injection at the second stage that allows the production of very monochromatic beams, and we discuss which kind of accelerator for the first stage will be needed in each case. For the second stage, we consider the case of a uniform plasma and the case of a plasma channel as well. The latter requires less energy than the first one: since the laser pulse is guided in the plasma channel, one does not have to rely on the Rayleigh length for exciting large amplitude plasma waves over a long distance. In practice, uniform long plasmas can be produced easily by focusing the laser beam onto a long gas cell, whereas long plasma channels can be produced using capillary discharges $[18,19]$.

To obtain stable working conditions, we limit the normalized laser amplitude to small values, namely, $a_{0} \approx$ $1-2$, avoiding nonlinear effects as self-focusing and other instabilities, and self-injection of plasma electrons. Plasma densities are in the range $n_{e} \approx 10^{16}-10^{17} \mathrm{~cm}^{-3}$. On one hand, low plasma densities imply long wake periods and thus less steep profiles of accelerating field. On the other hand, these densities allow us to attain the $\mathrm{GeV}$ range in one stage. The maximum energy gain limited by dephasing is of the order of tens of GeV.

Very small energy spreads can be obtained by injecting the electrons at the maximum of the longitudinal electric field. At this point, the derivative $d E_{z} / d z$ vanishes, and thus the variation of the electric field along the bunch length is minimal. Figure 3 shows the spatial distribution of electron density (top) and the longitudinal force on electrons (wakefield plus ponderomotive, bottom) for three laser and plasma conditions. In the first case [Fig. 3(a)], the normalized laser amplitude is $a_{0}=0.5$ and the plasma is homogeneous. For this relatively small value of $a_{0}$, the plasma responds almost linearly to the perturbation generated by the laser pulse. In this regime, wakes are straight, and thus, the maximum of longitudinal electric field separates regions of radial focusing fields from defocusing regions. Therefore, bunches centered at the maximum are not well trapped.

Trapping around the maximum of electric field requires bent wakes. Bent wakes are generated, for example, in inhomogeneous plasmas. In a parabolic plasma channel, there is a radial gradient of plasma wavelength associated with the gradient of plasma density. The plasma wavelength is largest at the axis where the density is smallest, thus resulting in a bent wake [Fig. 3(b)]. The maximum of longitudinal electric field no longer corresponds to the frontier between the focusing and defocusing region, i.e., there is a spatial window around this maximum where the radial electric field is focusing. A bunch injected inside this window will be well trapped and it will present the minimal final bandwidth.

Bent wakes can also be produced by using moderate intensity laser pulses. In this case, the bending is due to nonlinear effects. As $a_{0}$ grows, wakes become steeper and the wave front becomes curved due to the relativistic shifting of the plasma frequency [Fig. 3(c)]. We can see that the region of focusing field is much larger than in the linear homogeneous case, i.e. $\lambda p / 2$.

There are several alternatives for trapping electron bunches in the optimal region of largest field and smallest gradient. Figure 4 illustrates the main types of injection allowing the production of highly monochromatic beams. In the bottom of this figure, the longitudinal force in the first and second wake period is shown; several electron trajectories in the phase space $(\gamma, \xi)$ are shown in the top. 


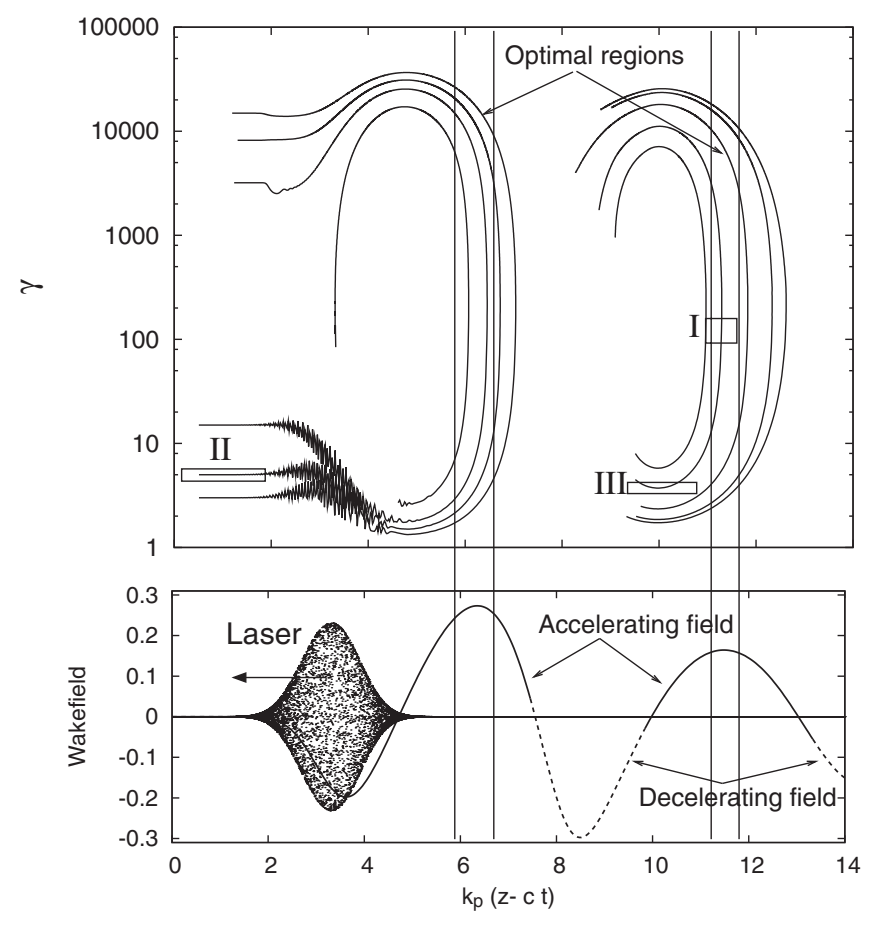

FIG. 4. Phase space $(\gamma, \xi)$ trajectories of electrons injected at the axis (top) and longitudinal force at the axis vs $\xi$ (bottom). The initial phase region of the different injection schemes (Ihigh energy, II-low energy before pulse, and III-low energy after pulse) are indicated with boxes.

Vertical lines indicate the limits of the optimal regions. Laser parameters are: $a_{0}=1, w_{0}=30 \mu \mathrm{m}, E=10 \mathrm{~J}$, and $P=0.16 \mathrm{PW}$. The pulse propagates in a parabolic plasma channel with $n_{0}=3 \times 10^{16} \mathrm{~cm}^{-3}$ perfectly matched.

Injection schemes can be divided in two groups, high energy schemes $\left(\gamma_{i} \approx \gamma_{p}\right)$ and low energy one $\left(\gamma_{i} \ll \gamma_{p}\right)$.

\section{A. Injection scenario: High electron energy case}

In the first type of injection, electrons are injected with relativistic energies in the optimal region (box I in Fig. 4). If electrons are extracted before the dephasing length, bunches will remain in the optimal region during all the acceleration process (trajectories are almost vertical in this region).

This kind of injection requires bunches much shorter than $\lambda_{p}$. On the other hand, the absolute energy spread is almost conserved, and therefore low final relative energy spreads can be obtained even with large initial relative energy spreads.

\section{B. Injection scenario: Low electron energy injection}

The second possibility is to inject electrons with subrelativistic energies, specifically with $\gamma_{i} \ll \gamma_{p}$, before the pulse (injection scheme II) or after the pulse (injection scheme III). Trajectories before the pulse are straight horizontal lines, because in this region there are no forces.
Because of this, electrons injected there with different phases but the same energy will follow the same trajectory. For a given range of energies, this trajectory will be in the optimal region, independently of the initial bunch length. The final energy spread will be finite due to the different times taken by the initial slipping and the initial finite energy spread. The second factor is in general the most significant because, in this case, absolute energy spread is not conserved. The disadvantage of this scheme is that charge is limited to values well below the $\mathrm{nC}$ because of the Coulomb repulsion between slow electrons.

The third possibility is to inject low energy electrons after the pulse (box III in Fig. 4). Trajectories in this region are not perfectly flat as before the pulse, and then shorter bunches are required. The main advantage is that the charge can be larger than in the case II, because, to some extent, the radial focusing force prevents the bunches from expanding radially. In any case, Coulomb repulsion prevents the focusing of electron bunches of some $\mathrm{MeV}$ and $\mathrm{nC}$ to radii of some microns, then these low energy schemes are restricted to low charges.

\section{A TWO STAGE LASER-PLASMA ACCELERATOR DESIGN}

In the first scheme presented, i.e., the high energy approach, the production of monoenergetic electron beams requires bunches much smaller than the plasma wave.

Plasma wave periods are typically in the $100 \mathrm{fs}$ range, therefore the duration of the injected electron bunch must be shorter than tens of fs. Such bunches are currently produced in laser-plasma accelerating structures. The technology for producing such a short bunch containing a high charge in conventional accelerators does not exist yet. On the other hand, the synchronization jitter between the pulse

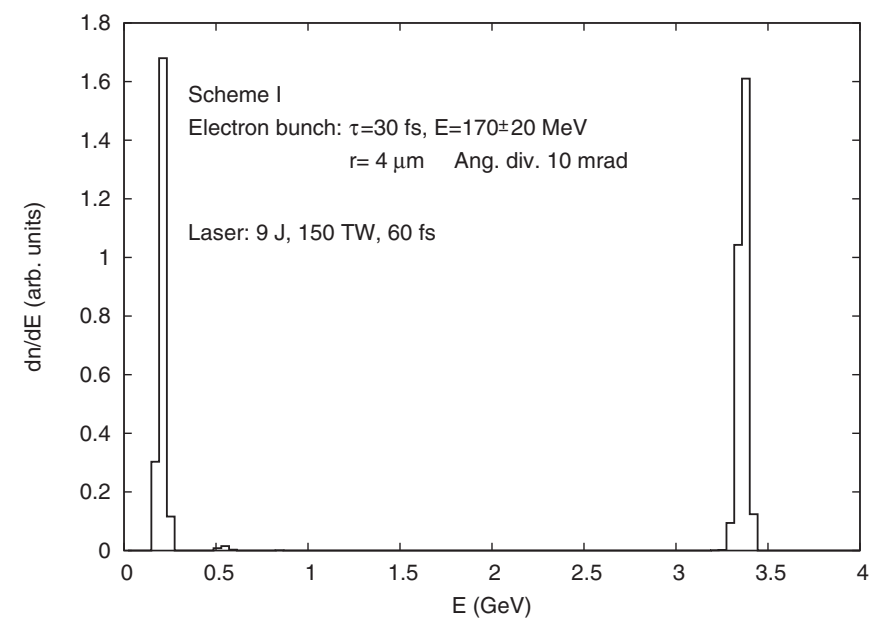

FIG. 5. Electron spectrum calculated for a $9 \mathrm{~J}-60 \mathrm{fs}-150 \mathrm{TW}$ laser guided in a parabolic plasma channel $n_{0}=1.1 \times$ $10^{17} \mathrm{~cm}^{-3}$. Acceleration distance $24 \mathrm{~cm}$. 

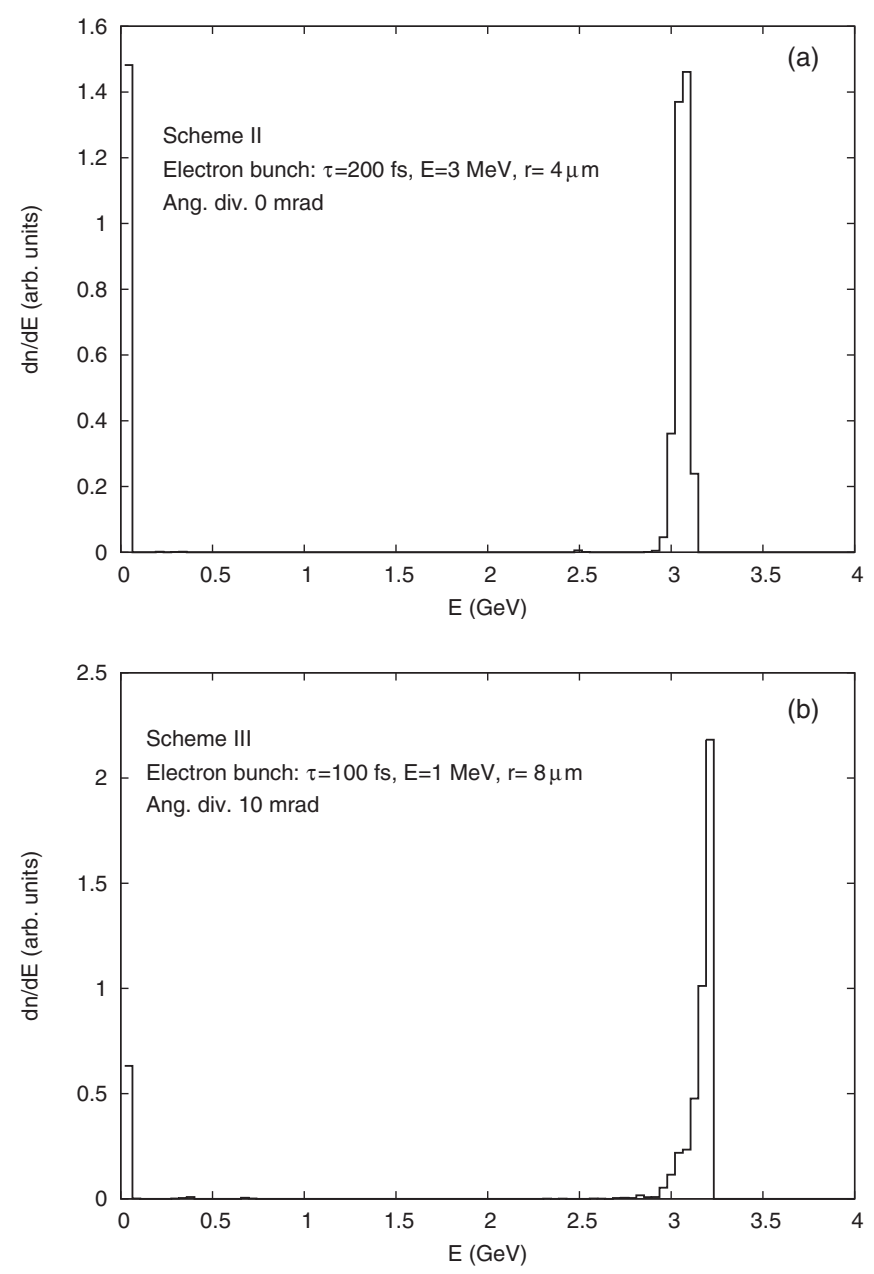

FIG. 6. Electron spectra for low injection energy schemes, before the pulse (a) and after the pulse (b) at the same laser and plasma parameters as those in Fig. 5.

and the electron bunch must be not larger than some fs. It is probably possible to achieve such a level of synchronization by using the same laser pulse for the first and second stage.

We performed numerical simulations of the acceleration of short bunches [20] using the code WAKE [21]. Electron bunch parameters are similar to those obtained in LOA experiments: mean energy of $170 \mathrm{MeV}$ with an energy spread FWHM of $40 \mathrm{MeV}$, and angular divergence of 10 mrad.

We study pulses guided in parabolic plasma channels with radial density profile given by $n(r)=n_{0}(1+$ $\left.0.585 r / r_{0}\right)$, with $n_{0}=1.1 \times 10^{17} \mathrm{~cm}^{-3}$ and $r_{0}=47 \mu \mathrm{m}$ and $24 \mathrm{~cm}$ long. The laser pulse duration is $60 \mathrm{fs}$, its energy, power, and maximum intensity are, respectively, $9 \mathrm{~J}, 150 \mathrm{TW}$, and $4.2 \times 10^{18} \mathrm{~W} \mathrm{~cm}^{-2}$. The bunches are injected in the optimal region of the first wake with a radius of $8 \mu \mathrm{m}$. Figure 5 shows the energy spectrum of the extracted bunch. We can see than roughly half of the electrons have been accelerated to $3.5 \mathrm{GeV}$, with a relative energy spread FWHM of $1 \%$ and an unnormalized emittance of $0.006 \mu \mathrm{m}$. The trapped fraction can be increased by reducing the initial bunch radius or by using wider pulses, which implies more laser energy.

Low injection energy schemes allow one to use much longer bunches. For example, Fig. 6 shows the energy spectra for a bunch injected before the pulse [scheme II, Fig. 6(a)] and after the pulse [scheme III, 6(b)], same laser and plasma parameters as those in Fig. 5. In the first case, the injected bunch has a duration of $200 \mathrm{fs}$, an energy of $3 \mathrm{MeV}$, radius $4 \mu \mathrm{m}$, and there is no angular divergence. As we can see, roughly half of the particles are trapped and accelerated in the wakefield, even for the small radius used. For larger radii or finite values of angular divergence, the trapped fraction drops drastically, because there is no radial focusing force before the pulse. In the second case [injection after the pulse, 6(b)], bunch parameters are: duration $100 \mathrm{fs}$, energy $1 \mathrm{MeV}$, radius $8 \mu \mathrm{m}$, and angular divergence $10 \mathrm{mrad}$. Almost all the electrons are trapped, but the spectrum is slightly less monochromatic. Conventional accelerator can be used in the first stage of these low energy schemes. On one hand, bunches can be as long as some hundreds of fs. On the other hand, sensibility to injection phase, especially in the scheme II, is weak compared with high energy schemes, then synchronization jitter is less critical.

\section{CONCLUSION}

The past few years have witnessed considerable progress in the generation and characterization of energetic particle beams with compact, short-pulse lasers. The laser-matter interaction mechanisms that are at play are not only fascinating physics but they also provide particle beams with unique properties, which make them remarkable scientific tools. These particle beams have already been successfully put to work in a number of experiments. The evolution of short-pulse laser technology, a field in rapid progress, will still improve the properties of these particle sources. For example, the development of diode pumped lasers will permit the increase of the laser power efficiency to values greater than $10 \%$, and it will also lead to a significant reduction of the size of such systems. The rapid evolution of CPA [22] based laser technology, coupled to progress in laser-plasma interaction modeling, will soon result in improved performance, lower cost, and still wider applicability of these compact particle sources. Numerical simulations show that multi-GeV electron beams with low energy spreads can be produced in a very compact way by injecting electron beams produced today in the bubble regime into a long plasma wave structure. Importantly, the beam quality is preserved as well as its very short duration. On a longer time scale, developments of these novel beam acceleration techniques should also be of interest for high energy physics experiments. 


\section{ACKNOWLEDGMENTS}

The authors acknowledge the support of the LOA laser team as well as the support of the European Community Research Infrastructure Activity under the FP6 "Structuring the European Research Area" program (CARE, Contract No. RII3-CT-2003-506395).

[1] T. Tajima and J.M. Dawson, Phys. Rev. Lett. 43, 267 (1979).

[2] F. Amiranoff et al., Phys. Rev. Lett. 81, 995 (1998).

[3] C. E. Clayton et al., Phys. Rev. Lett. 70, 37 (1993).

[4] M. J. Everett et al., Nature (London) 368, 527 (1994).

[5] V. Malka et al., Science 298, 1596 (2002).

[6] S. Fritzler et al., Phys. Rev. Lett. 92, 165006 (2004).

[7] S. P. D. Mangles et al., Nature (London) 431, 535 (2004).

[8] C. G. R. Geddes et al., Nature (London) 431, 538 (2004).
[9] J. Faure et al., Nature (London) 431, 541 (2004).

[10] A. Pukhov and J. Meyer-ter-Vehn, Appl. Phys. B 74, 355 (2002).

[11] Y. Glinec et al. (to be published).

[12] V. Malka, J. Faure, Y. Glinec, A. Pukhov, J. P. Rousseau, Phys. Plasmas 12, 056702 (2005).

[13] J. Faure et al., Phys. Rev. Lett. 95, 205003 (2005).

[14] J. Faure, Y. Glinec, G. Gallot, and V. Malka, Phys. Plasmas 13, 056706 (2006).

[15] J. Faure et al., Phys. Rev. Lett. 95, 205003 (2005).

[16] W. Lu et al. Nature Phys. (to be published).

[17] F. S. Tsung et al., Phys. Rev. Lett. 93, 185002 (2004).

[18] A. Zigler, J. Opt. Soc. Am. B 13, 68 (1996).

[19] S. M. Hooker et al., J. Opt. Soc. Am. B 17, 90 (2000).

[20] A. F. Lifschitz, J. Faure, V. Malka, and P. Mora, Phys. Plasmas 12, 093104 (2005).

[21] P. Mora and T. M. Antonsen, Phys. Plasmas 4, 217 (1997).

[22] D. Strikland and G. Mourou, Opt. Commun. 56, 219 (1985). 